\title{
COVID-19 and early-stage lung cancer both featuring ground-glass opacities: a propensity score-matched study
}

\author{
Ya-Jie Zhang $^{1 \#}$, Wen-Jie Yang ${ }^{2 \#}$, Dong Liu ${ }^{3 \#}$, Yu-Qin Cao ${ }^{1 \wedge}$, Yu-Yan Zheng ${ }^{1}$, Yi-Chao Han ${ }^{1}$, \\ Run-Sen Jin ${ }^{1}$, Yu Han ${ }^{1}$, Xiao-Yang Wang ${ }^{4}$, A-Shan Pan ${ }^{5}$, Jian-Yi Dai ${ }^{6}$, Qing-Feng Sun ${ }^{6}$, Feng-Quan Zhao ${ }^{7}$ \\ Qing-Yuan Yang ${ }^{3}$, Jia-Hao Zhang ${ }^{1}$, Sheng-Jun Liu ${ }^{8}$, Qian Da ${ }^{9}$, Wei Guo ${ }^{1}$, Cheng-Qiang Li ${ }^{1}$, \\ Wen-Tian Zhang ${ }^{1}$, Han Wu ${ }^{1}$, Xing-Shi Chen ${ }^{1}$, An-Qi Ji ${ }^{1}$, Jie Xiang ${ }^{1}$, Kai Chen ${ }^{1}$, Xi-Jia Feng ${ }^{1}$, \\ Xian-Fei Zhang ${ }^{1}$, Qi-Qi Cao ${ }^{2}$, Le Qin ${ }^{2}$, Jian Li $^{10}$, Min Zhou ${ }^{3}$, Yong Lu ${ }^{2}$, Chao-Fu Wang ${ }^{9}$, Fu-Hua Yan ${ }^{2}$, \\ He-Cheng $\mathrm{Li}^{1} \wedge$, Jie-Ming $\mathrm{Qu}^{3}$
}

${ }^{1}$ Department of Thoracic Surgery, ${ }^{2}$ Department of Radiology, ${ }^{3}$ Department of Respiratory and Critical Care Medicine, Ruijin Hospital, Shanghai Jiao Tong University School of Medicine, Shanghai, China; ${ }^{4}$ Department of Radiology, Ruian People's Hospital, Ruian, China; ${ }^{5}$ Department of Radiology, Yueqing People's Hospital, Yueqing, China; ${ }^{6}$ Department of Infectious Diseases, Ruian People's Hospital, Ruian, China; ${ }^{7}$ Department of Infectious Diseases, Wenzhou Central Hospital, Wenzhou, China; ${ }^{8}$ Department of Cardiology, ${ }^{9}$ Department of Pathology, ${ }^{10}$ Clinical Research Center, Ruijin Hospital, Shanghai Jiao Tong University School of Medicine, Shanghai, China

Contributions: (I) Conception and design: YJ Zhang, WJ Yang, FH Yan, HC Li, JM Qu; (II) Administrative support: FH Yan, HC Li, JM Qu, J Li, M Zhou, Y Lu, CF Wang; (III) Provision of study materials or patients: WJ Yang, XY Wang, AS Pan, JY Dai, QF Sun, FQ Zhao; (IV) Collection and assembly of data: YJ Zhang, D Liu, YQ Cao, YY Zheng, YC Han, JH Zhang, W Guo, CQ Li, WT Zhang, H Wu, XS Chen, AQ Ji, J Xiang, K Chen, XJ Feng, XF Zhang, QQ Cao, L Qin; (V) Data analysis and interpretation: YJ Zhang, WJ Yang, YQ Cao, RS Jin, YY Zheng, Y Han, SJ Liu, Q Da, CF Wang, J Li, M Zhou, Y Lu; (VI) Manuscript writing: All authors; (VII) Final approval of manuscript: All authors.

\#These authors contributed equally to this work.

Correspondence to: Professor Jie-Ming Qu. Department of Respiratory and Critical Care Medicine, Ruijin Hospital, Shanghai Jiao Tong University School of Medicine, 197 Rui Jin Er Road, Shanghai 200025, China. Email: jmqu0906@sina.cn; Professor He-Cheng Li. Department of Thoracic Surgery, Ruijin Hospital, Shanghai Jiao Tong University School of Medicine, 197 Rui Jin Er Road, Shanghai 200025, China. Email: lihecheng2000@hotmail.com; Professor Fu-Hua Yan. Department of Radiology, Ruijin Hospital, Shanghai Jiao Tong University School of Medicine, 197 Rui Jin Er Road, Shanghai 200025, China. Email: yanfuhua@yahoo.com.

Background: Radiological manifestations of coronavirus disease 2019 (COVID-19) featured ground-glass opacities (GGOs), especially in the early stage, which might create confusion in differential diagnosis with early lung cancer. We aimed to specify the radiological characteristics of COVID-19 and early lung cancer and to unveil the discrepancy between them.

Methods: One hundred and fifty-seven COVID-19 patients and 374 early lung cancer patients from four hospitals in China were retrospectively enrolled. Epidemiological, clinical, radiological, and pathological characteristics were compared between the two groups using propensity score-matched (PSM) analysis.

Results: COVID-19 patients had more distinct symptoms, tended to be younger $(\mathrm{P}<0.0001)$, male $(\mathrm{P}<0.0001)$, and had a higher body mass index $(\mathrm{P}=0.014)$. After 1:1 PSM, 121 matched pairs were identified. Regarding radiological characteristics, patients with a single lesion accounted for $17 \%$ in COVID-19 and $89 \%$ in lung cancer $(\mathrm{P}<0.0001)$. Most lesions were peripherally found in both groups. Lesions in COVID-19 involved more lobes (median 3.5 vs. $1 ; \mathrm{P}<0.0001$ ) and segments (median 6 vs. $1 ; \mathrm{P}<0.0001$ ) and tended to have multiple types (67\%) with patchy form (54\%). Early lung cancer was more likely to have a single type (92\%) with oval form (66\%). Also, COVID-19 and early lung cancer either had some distinctive features on computed tomography (CT) images.

Conclusions: Both COVID-19 and early lung cancers showed GGOs, with similar but independent features. The imaging characteristics should be fully understood and combined with epidemiological

^ ORCID: Yu-Qin Cao: 0000-0003-0127-7557; He-Cheng Li: 0000-0001-8069-6033. 
history, pathogen detection, laboratory tests, short-term CT reexamination, and pathological results to aid differential diagnosis.

Keywords: Coronavirus disease 2019 (COVID-19); lung neoplasms; ground glass opacity; radiology; propensity score

Submitted Jul 02, 2020. Accepted for publication Aug 10, 2020.

doi: $10.21037 /$ tlcr-20-892

View this article at: http://dx.doi.org/10.21037/tlcr-20-892

\section{Introduction}

Coronavirus disease 2019 (COVID-19) was caused by severe acute respiratory syndrome coronavirus 2 (SARS$\mathrm{CoV}-2$ ), and the epidemic was designated as a global health emergency by WHO on January 30, 2020 (1). As of June 20,2020 , there were more than $8,525,000$ reported cases with over 456,000 deaths globally (2). The pathogenesis of COVID-19 infection generally includes three stages. In the early stage, the virus infects the nasal cells through binding to angiotensin-converting enzyme 2 (ACE-2) and mounts a minor inflammatory response. Later, the virus propagates to the conducting respiratory airways, and the COVID-19 disease is clinically manifested at this time. Finally, the virus reaches the gas exchange units of the lung and infects alveolar type II cells. About $20 \%$ of the infected patients progresses to this stage and develops pulmonary infiltrates, with some of them developing very severe disease including frank lung injury and acute respiratory distress syndrome (ARDS) (3).With the rapid transmission of COVID-19, consensus guidelines should be steadily established and updated to prevent transmission and facilitate diagnosis and treatment (4).

Real-time polymerase chain reaction (RT-PCR) for SARS-CoV-2 nucleic acid test and virus gene sequencing is the current gold standard of diagnosis, but the timeconsuming procedures result in a prolonged diagnostic cycle (5). Remarkably, chest high-resolution computed tomography (HRCT) demonstrated a multiple-stage imaging evolution of COVID-19 (6). Most cases featured patchy/oval ground-glass opacities (GGOs) or consolidative pulmonary opacities on unenhanced computed tomography (CT) scans, while the enlargement and consolidation of GGO usually indicated the progression of pneumonia $(7,8)$. Aside from its pivotal role in evaluating the severity of pulmonary involvement in COVID-19, thoracic CT also serves as a significant supplement to discern false-negative patients undergoing the SARS-CoV-2 nucleic acid test, as these patients are likely to have typical CT findings (9).

Notably, low-dose CT (LDCT) is currently applied as an effective tool for screening early lung cancer, on which GGOs usually indicate potential malignant lesions $(10,11)$. The similarity of GGOs on CT images can create confusion in distinguishing COVID-19 from early lung cancer, especially in asymptomatic or nucleic acid negative patients. Also, there were reports on patients with early stage lung cancer combined COVID-19, presenting no symptoms of pneumonia. Some of them received surgery but experienced severe pneumonia and other serious complications or even death postoperatively (12). Thus, it is essential to distinguish these two diseases, which are both manifested as GGOs.

Herein we describe and characterize the essential radiological findings in a group of 157 patients with COVID-19 compared with a group of 374 patients with early stage lung cancer. To the best of our knowledge, this is the first analysis aiming to compare these two groups by accounting for confounding variables in attempt to minimize bias. Their epidemiological, clinical, and pathological characteristics are also evaluated in the hope of unveiling the discrepancy between these two diseases. We present the following article in accordance with the STROBE reporting checklist (http://dx.doi.org/10.21037/tlcr-20-892).

\section{Methods}

\section{Ethical approval}

The Ethics Commission of Ruijin Hospital approved this study (KY2020-26) and following the Helsinki Declaration (as revised in 2013). Written informed consent for this retrospective study was waived by the Ethics Commission of the designated hospital for emerging infectious diseases.

\section{Clinical specimen and data collection}

A total of 157 patients with COVID-19 confirmed by RT- 


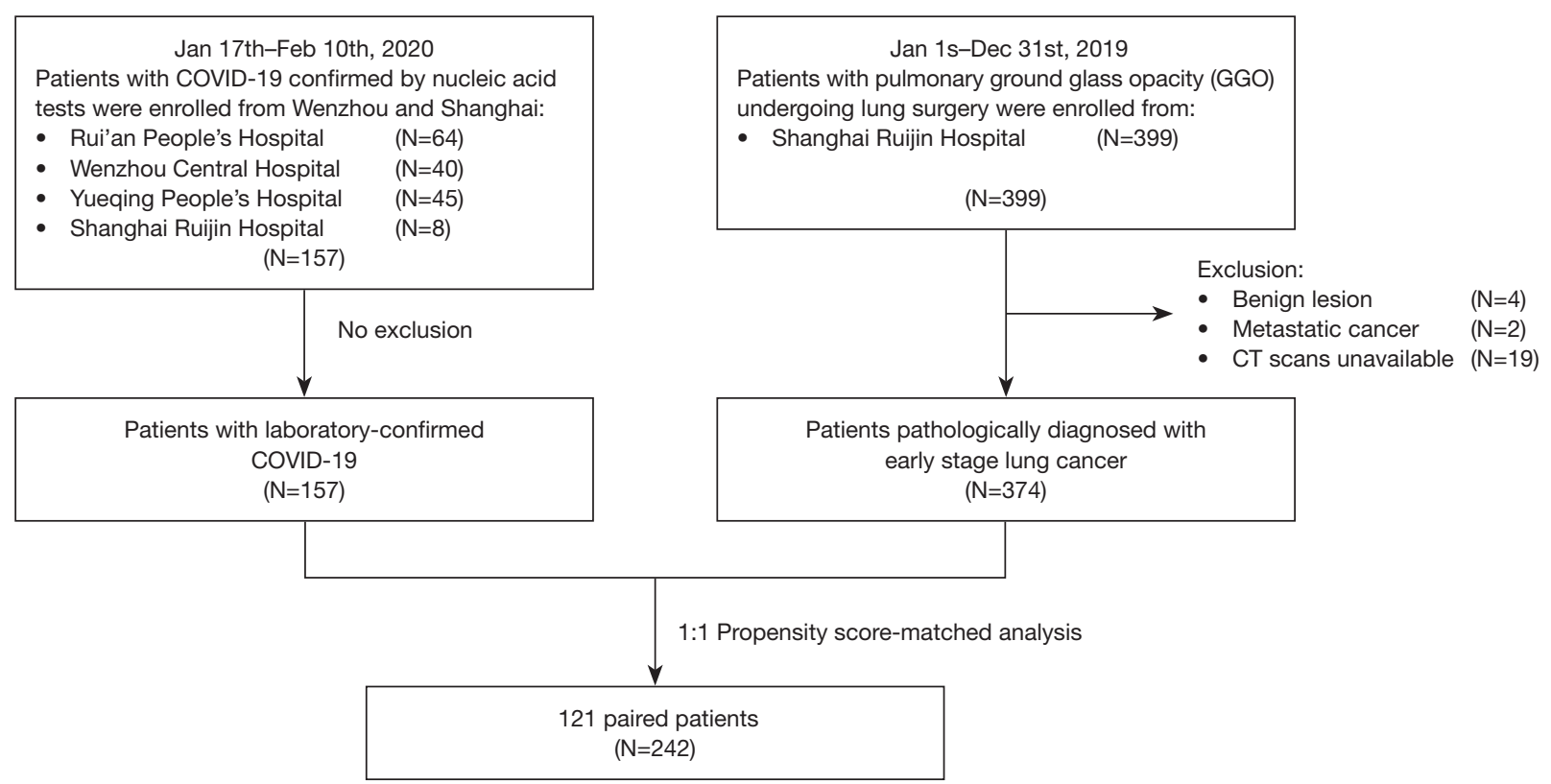

Figure 1 Diagram of patient selection. COVID-19, coronavirus disease 2019.

PCR from January 17 through February 10, 2020, were enrolled in this study, including eight patients from Ruijin Hospital Affiliated to Shanghai Jiao Tong University School of Medicine (Shanghai, China) and 149 patients from three tertiary hospitals in Wenzhou (Wenzhou, China). Meanwhile, we analyzed 374 patients admitted to Ruijin Hospital from January 1 through December 31, 2019, whose CT manifestations featured GGOs and whose postoperative pathology confirmed early stage lung cancer, as a control—the early lung cancer group (Figure 1).

The demographic data, epidemiological history, medical symptoms, laboratory tests, CT findings, and pathologic results were obtained from patients' medical records. For epidemiological and symptom data inaccessible from electronic medical records, the researchers communicated directly with patients or their families to substantiate.

\section{CT image review}

All CT examinations adhered to the common chest protocol: the patient was installed in a supine position with arms raised and held his/her breath during acquisition. For each patient, the following characteristics were evaluated (8): (I) type of lung lesions (pure GGO; mixed GGO; consolidation) (11); (II) form of lesions (oval; patchy); (III) distribution of lesions (unilateral lung; bilateral lung); (IV) number of involved lobes; (V) frequency of lobe involvement (right upper lobe; right middle lobe; right lower lobe; left upper lobe; left lower lobe); (VI) number of involved segments; (VII) frequency of segment involvement $\left(\mathrm{L} \mathrm{S}^{1+2} ; \mathrm{L} \mathrm{S}^{3} ; \mathrm{L} \mathrm{S}^{4} ; \mathrm{L} \mathrm{S}^{5} ; \mathrm{L} \mathrm{S}^{6} ; \mathrm{L} \mathrm{S}^{7+8} ; \mathrm{L} \mathrm{S}^{9} ; \mathrm{L} \mathrm{S}^{10} ; \mathrm{R} \mathrm{S}^{1} ; \mathrm{R} \mathrm{S}^{2}\right.$; $\mathrm{R} \mathrm{S}^{3} ; \mathrm{R} \mathrm{S}^{4} ; \mathrm{R} \mathrm{S}^{5} ; \mathrm{R} \mathrm{S}^{6} ; \mathrm{R} \mathrm{S}^{7}$; $\mathrm{R} \mathrm{S}^{8}$; $\mathrm{R} \mathrm{S}^{9} ; \mathrm{R} \mathrm{S}^{10}$ ); (VIII) other findings: air bronchogram, centrilobular nodules, tree-inbud, reticular pattern, subpleural linear opacity, bronchial dilation, cystic change, lobulated sign, pleural retraction, vessel convergence sign, lymphadenopathy, and pleural effusion.

Two fellowship-trained cardiothoracic radiologists with five years of experience reviewed all the CT scans through a viewing console independently. A third fellowship-trained cardiothoracic radiologist with 15 years of experience adjudicated a final decision when the two radiologists did not reach consensus.

\section{Statistical analysis}

To minimize the bias caused by the non-randomized selection of patients, we performed a propensity scorematched (PSM) analysis between two groups. Each patient's propensity score was calculated under a multivariable logistic regression model with covariates that showed significant difference at baseline (Table 1), including sex, age, body mass index (BMI), comorbidity of cardiocerebrovascular disease, digestive disease, and tumor history. 
Table 1 Demographic and clinical characteristics of patients

\begin{tabular}{|c|c|c|c|c|c|c|}
\hline \multirow[b]{2}{*}{ Variables } & \multicolumn{3}{|c|}{ Before PSM } & \multicolumn{3}{|c|}{ After PSM } \\
\hline & $\begin{array}{l}\text { COVID-19 } \\
(n=157)\end{array}$ & $\begin{array}{l}\text { Lung cancer } \\
\qquad(\mathrm{n}=374)\end{array}$ & $P$ value & $\begin{array}{l}\text { COVID-19 } \\
(n=121)\end{array}$ & $\begin{array}{l}\text { Lung cancer } \\
\qquad(n=121)\end{array}$ & $P$ value \\
\hline Male & $86(55 \%)$ & $131(35 \%)$ & & $58(48 \%)$ & $57(47 \%)$ & \\
\hline Female & $71(45 \%)$ & $243(65 \%)$ & & $63(52 \%)$ & $64(53 \%)$ & \\
\hline BMI $\left(\mathrm{kg} / \mathrm{m}^{2}\right)$, median (IQR) & 24 [21-26] & 23 [21-25] & 0.014 & 23 [21-25] & 23 [22-26] & 0.71 \\
\hline Exposure history, n (\%) & & & $<0.0001^{\dagger}$ & & & $<0.0001^{\dagger}$ \\
\hline Exposure to infected patient & $49(31 \%)$ & $0(0 \%)$ & & $36(30 \%)$ & $0(0 \%)$ & \\
\hline Recent travel to Wuhan & $84(54 \%)$ & $0(0 \%)$ & & $69(57 \%)$ & $0(0 \%)$ & \\
\hline Cardio-cerebrovascular disease & $29(18 \%)$ & $105(28 \%)$ & 0.027 & $25(21 \%)$ & $27(22 \%)$ & 0.88 \\
\hline Digestive disease & $8(5 \%)$ & $43(12 \%)$ & 0.034 & $8(7 \%)$ & $7(6 \%)$ & 1.00 \\
\hline Endocrine disease & $9(6 \%)$ & $32(9 \%)$ & 0.35 & $9(7 \%)$ & $5(4 \%)$ & 0.41 \\
\hline Neuropathy & $0(0 \%)$ & $6(2 \%)$ & 0.19 & $0(0 \%)$ & $2(2 \%)$ & 0.50 \\
\hline Respiratory disease & $3(2 \%)$ & $15(4 \%)$ & 0.34 & $2(2 \%)$ & $4(3 \%)$ & 0.68 \\
\hline Tumor history & $2(1 \%)$ & $28(7 \%)$ & 0.0087 & $2(2 \%)$ & $2(2 \%)$ & 1.00 \\
\hline \multicolumn{7}{|l|}{ Symptoms, n (\%) } \\
\hline Fever & $122(78 \%)$ & $2(1 \%)$ & $<0.0001$ & $99(82 \%)$ & $0(0 \%)$ & $<0.0001$ \\
\hline Chest distress & $16(10 \%)$ & $3(1 \%)$ & $<0.0001$ & $13(11 \%)$ & $3(2 \%)$ & 0.020 \\
\hline Muscle soreness & $8(5 \%)$ & $0(0 \%)$ & $<0.0001$ & $4(3 \%)$ & $0(0 \%)$ & 0.12 \\
\hline Diarrhea & $12(8 \%)$ & $0(0 \%)$ & $<0.0001$ & $7(6 \%)$ & $0(0 \%)$ & 0.014 \\
\hline No obvious symptoms & $5(3 \%)$ & $344(92 \%)$ & $<0.0001$ & $2(2 \%)$ & $110(91 \%)$ & $<0.0001$ \\
\hline \multicolumn{7}{|c|}{ Laboratory findings at admission, median (IQR) } \\
\hline White blood cell (G/L) & $4.6(3.6-6.0)$ & $5.1(4.3-6.0)$ & 0.00027 & $4.5(3.3-5.7)$ & $5.4(4.5-6.4)$ & $<0.0001$ \\
\hline Neutrophil (G/L) & $2.64(1.99-3.90)$ & $2.81(2.30-3.60)$ & 0.16 & $2.60(1.80-3.90)$ & $3.07(2.40-3.60)$ & 0.032 \\
\hline Lymphocyte (G/L) & $1.29(0.98-1.65)$ & $1.70(1.39-2.00)$ & $<0.0001$ & $1.19(0.90-1.50)$ & $1.74(1.50-2.10)$ & $<0.0001$ \\
\hline Alanine aminotransferase (U/L) & $20.0(14.0-33.0)$ & $19.0(14.0-26.0)$ & 0.11 & $18.5(13.3-32.0)$ & $19.0(14.0-29.0)$ & 0.85 \\
\hline Aspartate aminotransferase (U/L) & $23.0(19.0-34.5)$ & $20.0(17.0-25.0)$ & $<0.0001$ & $23.0(19.0-33.5)$ & $20.0(16.0-24.0)$ & $<0.0001$ \\
\hline $\mathrm{D}$-dimer (mg/L) & $0.25(0.18-0.52)$ & $0.24(0.19-0.37)$ & 0.29 & $0.27(0.19-0.63)$ & $0.21(0.17-0.32)$ & 0.017 \\
\hline Viral nucleic acid detection, n (\%) & $157(100 \%)$ & $N A^{\S}$ & NA & $121(100 \%)$ & $N A^{\S}$ & NA \\
\hline
\end{tabular}

${ }^{\dagger}$, Fisher's exact test for overall distribution; ${ }^{\S}$, viral nucleic acid detection was not applicable (NA) in patients with lung cancer. PSM, propensity score-matched; COVID-19, coronavirus disease 2019; IQR, interquartile range. 
Patients in the COVID-19 group were matched 1:1 with patients in the early lung cancer group using the nearestneighbor method with a caliper of 0.02 .

Continuous variables were presented as median [interquartile range (IQR)] and compared by Wilcoxon rank-sum test between the groups. Pearson's Chi-squared test or Fisher's exact test was applied for comparing categorical variables. PSM and statistical analyses were performed by using R software (version 3.5.3, R Foundation for Statistical Computing, Vienna, Austria). A P value less than 0.05 was considered statistically significant.

\section{Role of the funding source}

The funders of this study had no role in study design, data collection, data analysis, data interpretation, writing of the manuscript, or decision to submit the article for publication.

\section{Results}

\section{Demographic and clinical characteristics}

A total of 157 patients with confirmed COVID-19 and 374 early stage lung cancer patients were included in this study. Patients in the COVID-19 group tended to be younger [median 46 years (IQR 36-55 years) vs. 58 years (IQR 49-65 years); $\mathrm{P}<0.0001]$, male sex $(55 \%$ vs. $35 \% ; \mathrm{P}<0.0001$ ), and with higher BMI [median $24 \mathrm{~kg} / \mathrm{m}^{2}$ (IQR $21-26 \mathrm{~kg} / \mathrm{m}^{2}$ ) vs. $23 \mathrm{~kg} / \mathrm{m}^{2}$ (IQR $21-25 \mathrm{~kg} / \mathrm{m}^{2}$ ); $\left.\mathrm{P}=0.014\right]$. The incidence of comorbidities including cardio-cerebrovascular disease ( $18 \%$ vs. $28 \% ; \mathrm{P}=0.027$ ), digestive disease ( $5 \%$ vs. $12 \%$; $\mathrm{P}=0.034)$, and tumor history ( $1 \%$ vs. $7 \% ; \mathrm{P}=0.0087)$ were significantly lower in COVID-19 patients. After PSM analysis controlling for sex, age, BMI, and comorbidities, 121 cases from each group were matched (Figure 1). As expected, this resulted in similar distributions of propensity scores (Figure S1). The demographic and clinical data of two groups before and after PSM analysis were presented in Table 1.

Before PSM, most of the patients $(138 / 157,88 \%)$ with COVID-19 had obvious exposure history, either exposed to infected patient or having recent travel to Hubei Province. Patients with COVID-19 had significantly higher incidences of various pneumonia symptoms than lung cancer patients, including fever $(78 \%$ vs. $1 \% ; \mathrm{P}<0.0001)$, cough $(59 \%$ vs. $5 \% ; \mathrm{P}<0.0001)$, sputum $(32 \%$ vs. $2 \%$; $\mathrm{P}<0.0001)$, dyspnea $(2 \%$ vs. $0 \% ; \mathrm{P}=0.026)$, snot $(4 \%$ vs. $0 \% ; \mathrm{P}=0.00062)$, chest distress $(10 \%$ vs. $1 \% ; \mathrm{P}<0.0001)$, muscle soreness ( $5 \%$ vs. $0 \% ; \mathrm{P}<0.0001)$, and diarrhea $(8 \%$ vs. $0 \% ; \mathrm{P}<0.0001)$. For laboratory findings at admission, COVID-19 patients had significantly lower counts of white blood cell [median 4.6 G/L (IQR 3.6-6.0 G/L) vs. 5.1 G/L (IQR 4.3-6.0 G/L); P=0.00027] and lymphocyte count [median 1.29 G/L (IQR 0.98-1.65 G/L) vs. $1.70 \mathrm{G} / \mathrm{L}$ (IQR 1.39-2.00 G/L); $\mathrm{P}<0.0001]$, and significantly higher aspartate aminotransferase [median 23.0 U/L (IQR 19.0$34.5 \mathrm{U} / \mathrm{L}$ ) vs. 20.0 U/L (IQR 17.0-25.0 U/L); $\mathrm{P}<0.0001]$ compared with lung cancer patients. Neutrophil count, alanine aminotransferase, and D-dimer revealed no significant difference between two groups before PSM.

After PSM, the difference of exposure history, incidence of symptoms, and laboratory findings were consistent. However, the incidence of dyspnea $(\mathrm{P}=0.50)$, snot $(\mathrm{P}=0.060)$, and muscle soreness $(\mathrm{P}=0.12)$ between two groups were no longer significantly different, whereas neutrophil count [median 2.60 G/L (IQR 1.80-3.90 G/L) vs. 3.07 G/L (IQR 2.40-3.60 G/L); $\mathrm{P}=0.032$ ] and D-dimer [median $0.27 \mathrm{mg} / \mathrm{L}$ (IQR 0.19-0.63 mg/L) vs. $0.21 \mathrm{mg} / \mathrm{L}$ (IQR $0.17-0.32 \mathrm{mg} / \mathrm{L}$ ); $\mathrm{P}=0.017]$ reached statistical difference.

\section{Radiological findings}

By reviewing CT scans, 17 COVID-19 patients had negative radiological results. A total of 1,207 lung lesions were found in the 140 COVID-19 patients, and 448 lesions were found in the 374 lung cancer patients. Peripheral lesions predominated the location in both groups (COVID-19, 923/1,207 lesions, 76\%; lung cancer, 333/448 lesions, 74\%). In the lesions of COVID-19 patients, the proportion of pure GGO, mixed GGO, and consolidation were $27 \%, 58 \%$, and $14 \%$, respectively. In the lesions of lung cancer patients, the correspondent proportions were $46 \%, 47 \%$, and $5 \%$ (Table S1).

Table 2 presented the quantitative comparison of CT findings between two groups. The number, type, form, distribution of lesions, and its specific features achieved comparable results before and after PSM. Among the matched groups, 21/121 (17\%) of the COVID-19 patients and 108/121 (89\%) of the lung cancer patients presented with a single lesion $(\mathrm{P}<0.0001)$. Patients with COVID-19 had more involved lobes [median 3.5 (IQR 2-5) vs. 1 (IQR $1-1) ; \mathrm{P}<0.0001$; Figure $2 A]$ and more involved segments [median 6 (IQR 2-13) vs. 1 (IQR 1-1); $\mathrm{P}<0.0001$; Figure 2B] compared with lung cancer patients. The specific distribution of involved lobes and segments after PSM was showed in Figure 2C,D. Most patients with COVID-19 
Table 2 Radiological findings of patients

\begin{tabular}{|c|c|c|c|c|c|c|}
\hline \multirow[b]{2}{*}{ Variables } & \multicolumn{3}{|c|}{ Before PSM } & \multicolumn{3}{|c|}{ After PSM } \\
\hline & $\begin{array}{l}\text { COVID-19 } \\
(n=157)\end{array}$ & $\begin{array}{l}\text { Lung cancer } \\
\qquad(\mathrm{n}=374)\end{array}$ & $P$ value & $\begin{array}{c}\text { COVID-19 } \\
(n=121)\end{array}$ & $\begin{array}{l}\text { Lung cancer } \\
\qquad(n=121)\end{array}$ & $P$ value \\
\hline $\begin{array}{l}\text { Number of involved lobes, } \\
\text { median }[\mathrm{IQR}]\end{array}$ & $4[2-5]$ & $1[1-1]$ & $<0.0001$ & $3.5[2-5]$ & $1[1-1]$ & $<0.0001$ \\
\hline Type of lesions, $n(\%)$ & & & $0.00050^{\dagger}$ & & & $0.00050^{\dagger}$ \\
\hline Pure GGO & $6(4 \%)$ & $156(42 \%)$ & $<0.0001$ & $1(1 \%)$ & $61(50 \%)$ & $<0.0001$ \\
\hline Mixed GGO & 27 (17\%) & $175(47 \%)$ & $<0.0001$ & $25(21 \%)$ & $49(40 \%)$ & 0.0013 \\
\hline Mixed GGO \& consolidation & $16(10 \%)$ & $5(1 \%)$ & $<0.0001$ & $14(12 \%)$ & $2(2 \%)$ & 0.0044 \\
\hline Pure \& Mixed \& consolidation & $32(20 \%)$ & $0(0 \%)$ & $<0.0001$ & $27(22 \%)$ & $0(0 \%)$ & $<0.0001$ \\
\hline No lesions & $17(11 \%)$ & $0(0 \%)$ & $<0.0001$ & $9(7 \%)$ & $0(0 \%)$ & 0.0033 \\
\hline Form of lesions, n (\%) & & & $<0.0001^{\S}$ & & & $<0.0001^{\dagger}$ \\
\hline Oval & $7(4 \%)$ & $236(63 \%)$ & $<0.0001$ & $6(5 \%)$ & $80(66 \%)$ & $<0.0001$ \\
\hline Patchy & $77(49 \%)$ & $112(30 \%)$ & $<0.0001$ & $65(54 \%)$ & $36(30 \%)$ & 0.00026 \\
\hline Oval \& patchy & $56(36 \%)$ & $26(7 \%)$ & $<0.0001$ & $41(34 \%)$ & $5(4 \%)$ & $<0.0001$ \\
\hline No lesions & $17(11 \%)$ & $0(0 \%)$ & $<0.0001$ & $9(7 \%)$ & $0(0 \%)$ & 0.0033 \\
\hline Pleural effusion, n (\%) & $11(7 \%)$ & $1(0 \%)$ & $<0.0001$ & $10(8 \%)$ & $1(1 \%)$ & 0.014 \\
\hline \multicolumn{7}{|l|}{ Other features, n (\%) } \\
\hline Air bronchogram & $88(56 \%)$ & $52(14 \%)$ & $<0.0001$ & $73(60 \%)$ & $13(11 \%)$ & $<0.0001$ \\
\hline Centrilobular nodules & $6(4 \%)$ & $0(0 \%)$ & 0.00062 & $5(4 \%)$ & $0(0 \%)$ & 0.060 \\
\hline Tree-in-bud & $2(1 \%)$ & $0(0 \%)$ & 0.087 & $1(1 \%)$ & $0(0 \%)$ & 1.00 \\
\hline Reticular pattern & $86(55 \%)$ & $0(0 \%)$ & $<0.0001$ & $73(60 \%)$ & $0(0 \%)$ & $<0.0001$ \\
\hline Subpleural linear opacity & $32(20 \%)$ & $0(0 \%)$ & $<0.0001$ & $29(24 \%)$ & $0(0 \%)$ & $<0.0001$ \\
\hline Bronchial dilatation & $27(17 \%)$ & $5(1 \%)$ & $<0.0001$ & $23(19 \%)$ & $0(0 \%)$ & $<0.0001$ \\
\hline Cystic change & $12(8 \%)$ & $80(21 \%)$ & 0.00022 & $8(7 \%)$ & $20(17 \%)$ & 0.027 \\
\hline Lobulated sign & $0(0 \%)$ & $90(24 \%)$ & $<0.0001$ & $0(0 \%)$ & $25(21 \%)$ & $<0.0001$ \\
\hline Pleural retraction & $0(0 \%)$ & $86(23 \%)$ & $<0.0001$ & $0(0 \%)$ & $24(20 \%)$ & $<0.0001$ \\
\hline Vessel convergence sign & $0(0 \%)$ & $16(4 \%)$ & 0.0045 & $0(0 \%)$ & $3(2 \%)$ & 0.25 \\
\hline
\end{tabular}

${ }^{\dagger}$, Fisher's exact test for overall distribution; ${ }^{\S}$, Pearson's chi-squared test for overall distribution. PSM, propensity score-matched; COVID-19, coronavirus disease 2019; IQR, interquartile range; GGO, ground-glass opacity. 


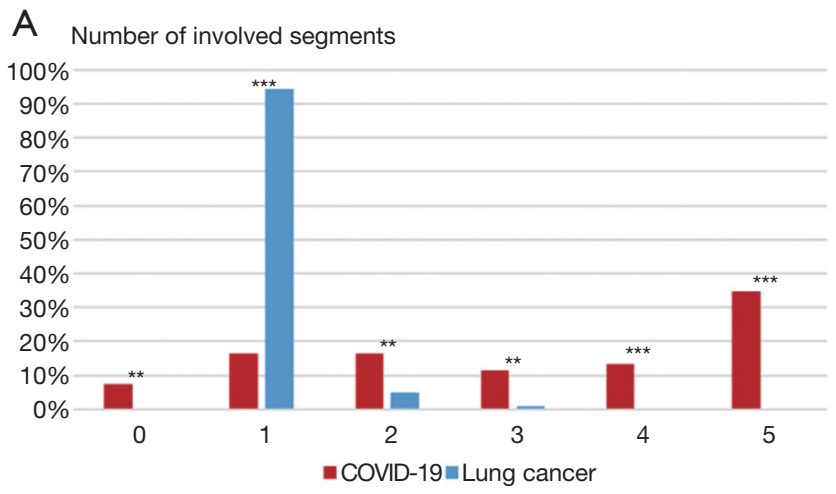

B Number of involved lobes
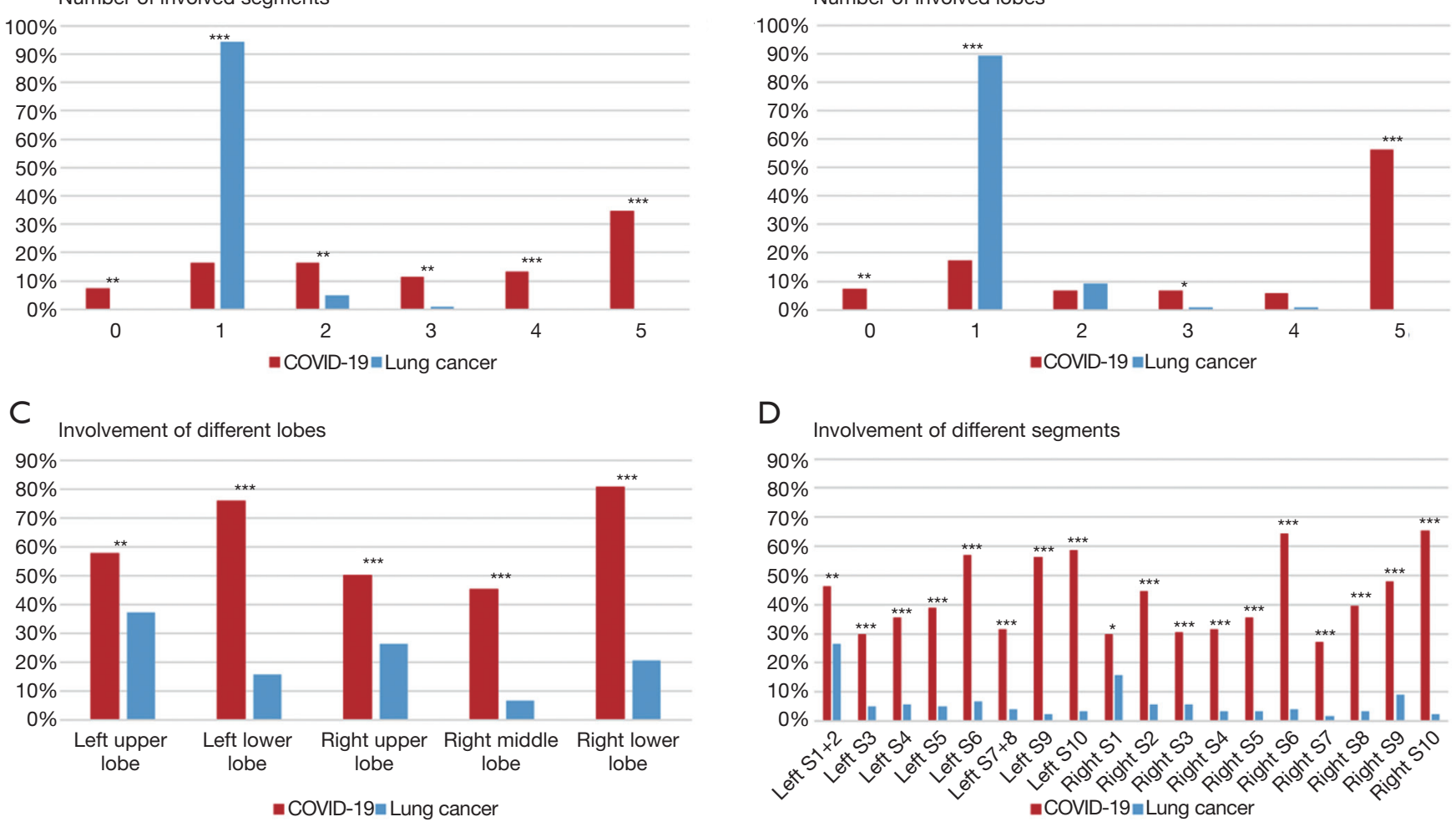

Figure 2 Involvement of different lobes and segments. COVID-19, coronavirus disease 2019.

(81/121, 67\%) had more than one type of lung lesions, while lung cancer patients tended to have either pure GGO (61/121, $50 \%)$ or mixed GGO (49/121, 40\%). Regarding the form, COVID-19 presented more patchy lesions (65/121, 54\%), while lung cancer presented more oval lesions (80/121, 66\%). Most patients with COVID-19 had lesions bilaterally $(89 / 121,74 \%)$, while most cancer patients had unilateral lung lesions (117/121, 97\%). Lymphadenopathy and pleural effusion were observed in 5/121 (4\%) and 10/121 (8\%) patients with COVID-19, respectively. Among the matched cancer patients, no one presented with lymphadenopathy, and only one patient $(1 / 121,1 \%)$ presented with pleural effusion on the CT scan. Air bronchogram and cystic change were observed in both groups. Air bronchogram was significantly more frequent in COVID-19 patients $(60 \%$ vs. $11 \% ; \mathrm{P}<0.0001)$, while the cystic change was less frequent in the COVID-19 group (7\% vs. 17\%; $\mathrm{P}=0.027)$. COVID-19 was also featured by reticular pattern $(73 / 121,60 \%)$, subpleural linear opacity (29/121, 24\%), bronchial dilatation (23/121, 19\%), centrilobular nodules $(5 / 121,4 \%)$, and tree-in-bud (1/121, $1 \%)$, whereas none of these signs were observed on the CT scans of matched cancer patients. On the contrary, lobulated sign $(25 / 121,21 \%)$, pleural retraction $(24 / 121,20 \%)$, and vessel convergence sign $(3 / 121,2 \%)$ were present in lung cancer patients but absent in those with COVID-19.

\section{Pathological results}

There is currently insufficient pathologic data on the COVID-19 from autopsy or biopsy, especially its comparison with early lung cancer presenting as GGO. However, a patient with an irregular solid nodule in the right middle lobe and bilateral ground-glass lesions underwent right middle lobectomy, and the specimen was found to have been infected by SARS-CoV-2 (12). The pathology indicated that the early phase of pulmonary pathological change of COVID-19 might be presented as edema, prominent globular proteinaceous exudate (Figure $S 2 A$ ), scattered spherical secretions or globules (Figure $S 2 B$ ), patchy inflammatory infiltration of mononuclear cells and multinucleated giant cells (Figure S2C), and focal hyperplasia of pneumocytes with interstitial thickening (Figure S2D). Meanwhile, neutrophil infiltration and hyaline 
membranes were not prominent. Occasionally, suspected viral inclusions might also be noted in some of these cells (Figure $S 2 D$ ).

Regarding early stage lung cancer, adenocarcinoma in situ (AIS), minimally invasive adenocarcinoma (MIA), and surrounding pulmonary tissue could coexist (Figure S2E). Yet to COVID-19, the morphological feature of AIS was usually presented by tumor cells growing along the alveolar wall without cellular space, and most cells were arranged in a single layer with moderate density (Figure $S 2 F$ ). MIA glands intruded into the stroma at acute angles and triggered focal connective tissue reactive hyperplasia (Figure $S 2 G$ ). Another feature of adenocarcinoma was the increased cellular dysplasia, including enlarged nuclei, thicker chromatin, and pseudo-nuclear inclusions (Figure S2H).

\section{Discussion}

The ongoing outbreak of novel coronavirus has infected hundreds of thousands of people in China and other countries worldwide. One of the most challenging problems is to ensure diagnostic sensitivity and specificity. In addition to the gold standard of nucleic acid test, CT is a practical and indispensable auxiliary examination for the diagnosis of COVID-19. Moreover, it is possible to discover COVID-19 despite negative nucleic acid levels $(13,14)$. Meanwhile, lung cancer is the most common malignancy around the world, mostly at the early stage (15). Enormous demand is called for to distinguish COVID-19 from early stage lung cancer during this public health emergency. Among the CT scans featured as GGO, attenuation was increased without covering up bronchial blood vessels, which showed focal damage of alveolar. The special imaging manifestation was paralleled with the pathological findings such as alveolar edema and proteinaceous exudates, where prominent inspissated spherical secretions, vascular congestion and multinucleated giant cells within the airspaces were seen. Moreover, proliferation of pneumocyte and interstitial fibroblasts decreased alveolar cavity (12). GGO predominates the radiological features of both COVID-19 (16) and early stage lung cancer $(17,18)$. Both diseases could show unilateral or bilateral, oval, or patchy GGO as single or multiple lesions on CT scans, which poses challenges to the discrimination, especially for clinically suspected COVID-19 cases with negative nucleic acid results. As shown in Figure 3, the two exemplified GGO lesions could hardly tell the difference between COVID-19 and early stage lung cancer on CT images. Unfortunately, the similarity could cheat the surgeons by GGO-featured COVID-19 lesions and result in inappropriate operations, where pathology eventually confirmed adenocarcinoma combined with SARS-CoV-2 infection.

Difficulties do exist, but we have found some characteristics of differentiation. This study reveals that COVID-19 predominates bilateral (74\%), patchy (54\%) GGOs, while early stage lung cancer predominates unilateral (97\%), oval (66\%) GGOs. The tendency of shape and distribution is consistent with previous reports $(19,20)$. More lobes are involved in the COVID-19 group than in the lung cancer group, like the results from other papers $(7,19)$. Specific radiographic patterns might help with distinction. COVID-19 is more featured by air bronchogram, reticular pattern, subpleural linear opacity, bronchial dilatation, centrilobular nodules, and tree-in-bud; on the contrary, lobulated sign, pleural retraction, cystic change, and vessel convergence sign are mostly present in lung cancer patients (Figure 4). Also, the rapid progression of COVID-19 on CT offers a dynamic reference for diagnosis. The initial phase of COVID-19, i.e., zero to four or five days, witnesses a classical GGO characteristic with occasional consolidation $(6,19)$. The lesion grows in size as the disease progresses around five days later (Figure 5), and additional manifestations appear including crazy-paving pattern, increased consolidation, enlarged fibrous stripe, and reverse halo sign $(7,19)$. In lung cancer patients, the growth of GGOs is associated with increasing malignancies (21). However, these changes are preferably indolent with an extremely long doubling time, particularly in pure GGOs (median, 1,200 days) (22). Therefore, yet to COVID-19, the dynamic evolution of early lung cancer on CT is merely expected during a brief follow-up period (Figure 5).

The above differences in CT images are subtle but of excellent value to differentiate the two entities. However, COVID-19 diagnosis requires a comprehensive evaluation combined with clinical symptoms, epidemiological investigation, and laboratory tests. Patients with manifestations of fever, cough, shortness of breath, and myalgia should be emphasized (23). Travel history to Wuhan or other epidemic areas and close contacts with COVID-19 patients raise suspicion of infection. Above all, real-time RT-PCR analysis of nucleic acid remains the key to a definite diagnosis (24).

It is necessary but also clinically significant to illustrate the fine distinction of CT imaging between COVID-19 and early lung cancer. Identification of GGO indicating COVID-19 by chest CT could discover those asymptomatic (25) 

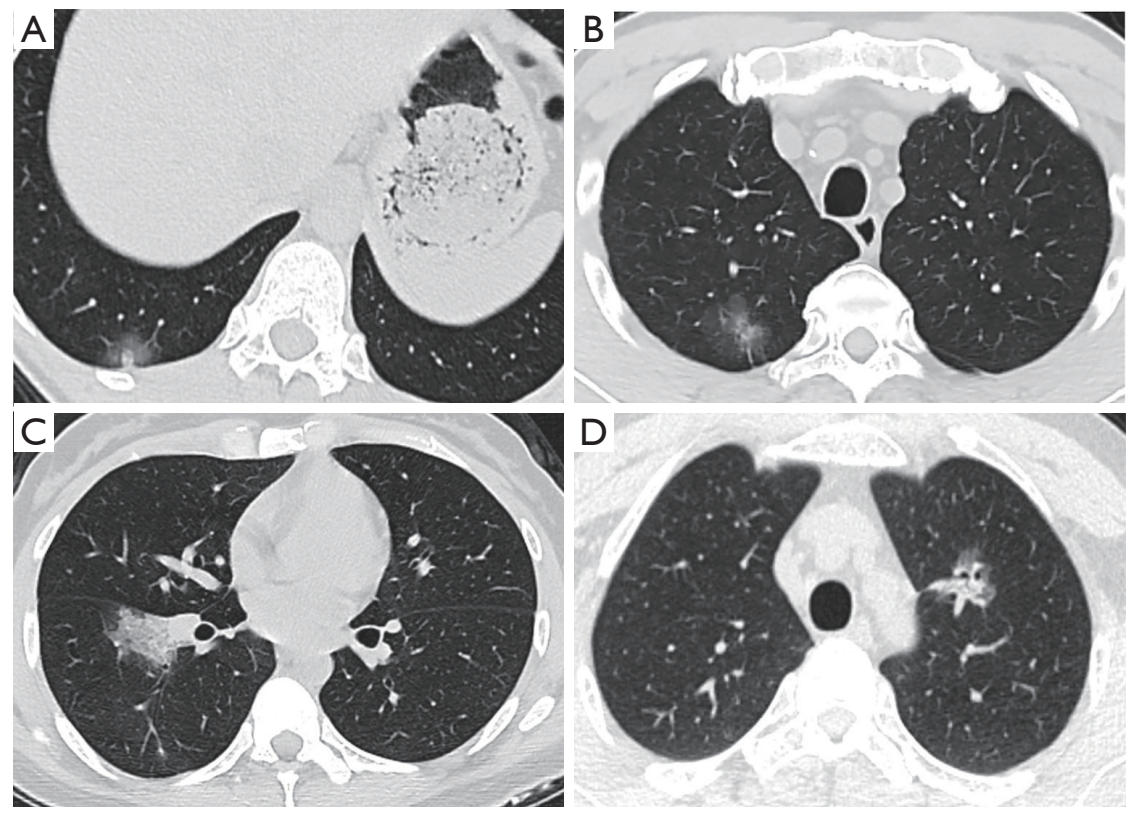

Figure 3 CT scans with similar manifestations between COVID-19 and lung cancer. Lesions in (A) and (B) were both presented as GGO: (A) CT scan of a patient diagnosed with COVID-19; (B) CT scan of a patient diagnosed with lung cancer. Lesions in (C) and (D) were both presented as patchy opacification: (C) CT scan of a patient diagnosed with COVID-19; (D) CT scan of a patient diagnosed with lung cancer. COVID-19, coronavirus disease 2019; GGO, ground-glass opacity.

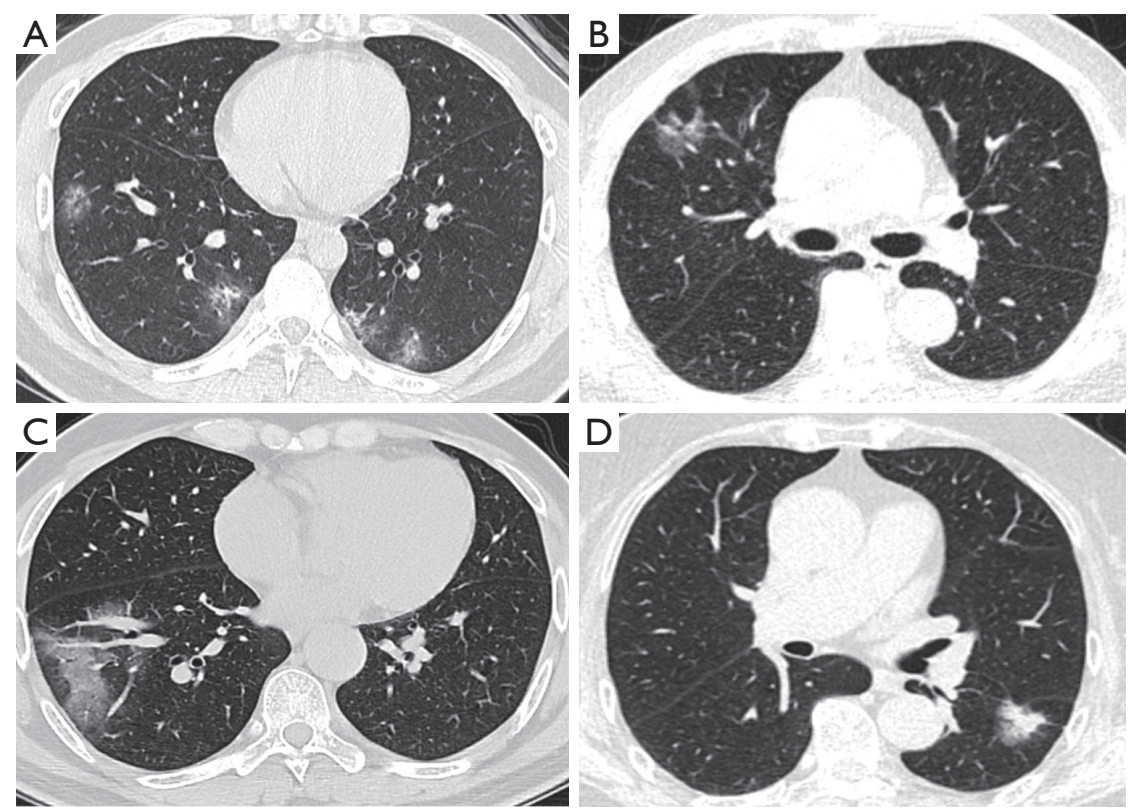

Figure 4 CT scans with distinct manifestations between COVID-19 and lung cancer. Lesions in (A) and (B) were both presented as GGO: (A) CT scan of a patient diagnosed with COVID-19, without pleural retraction or vessel convergence sign; (B) CT scan of a patient diagnosed with lung cancer, with pleural retraction and cystic change. Lesions in (C) and (D) were both presented as patchy opacification: (C) CT scan of a patient diagnosed with COVID-19 presenting irregular shape on CT, without pleural retraction; (D) CT scan of a patient diagnosed with lung cancer, with pleural retraction, lobulated sign, and spiculate protuberance. GGO, ground-glass opacity; COVID-19, coronavirus disease 2019. 


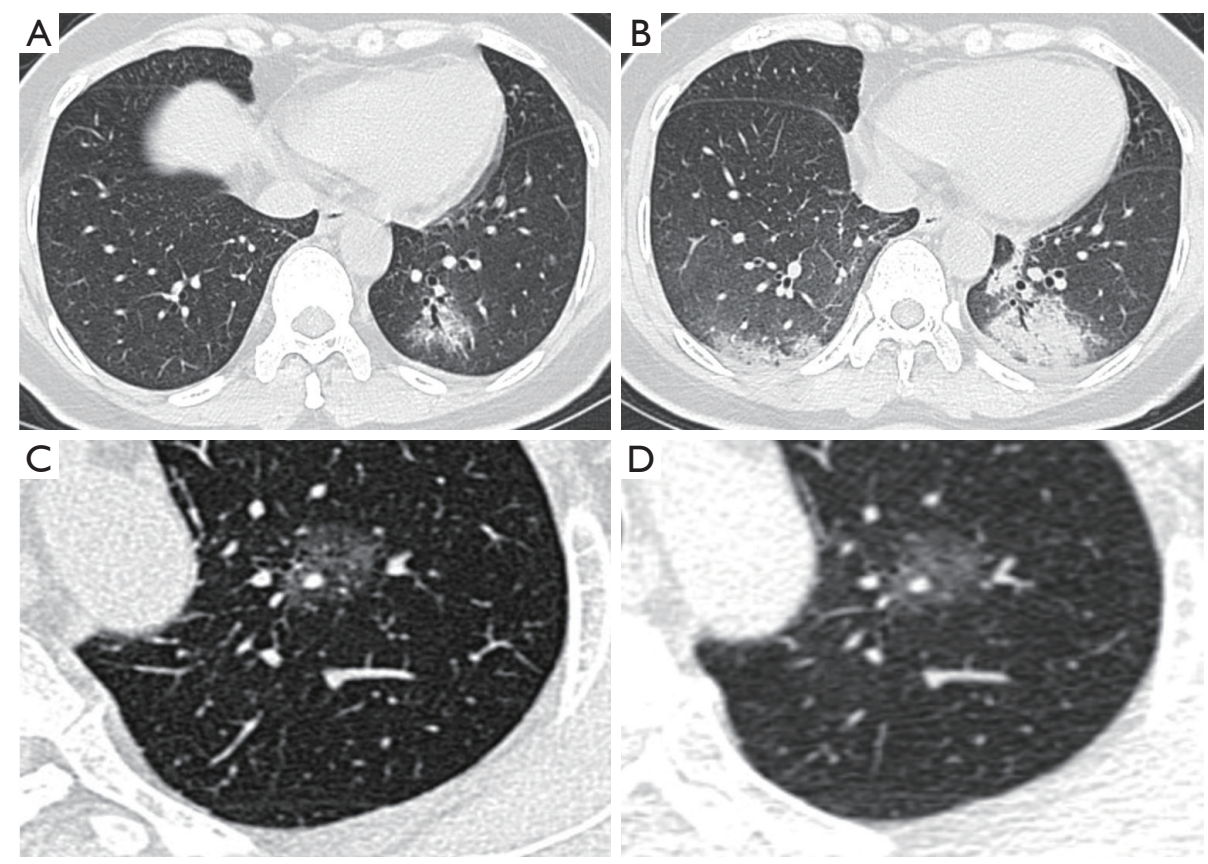

Figure 5 Progression of GGOs in COVID-19 and lung cancer. Panels (A) and (B) showed typical evolution on CT scans of a patient diagnosed with COVID-19 who presented with the only cough as a symptom: (A) on day 4; a mixed GGO was demonstrated in the left lower lobe with air bronchogram; (B) on day 11, the primary lesion was significantly enlarged with consolidation. What is more, there was a new area of subpleural patchy opacification in the right lower lobe. Panels (C) and (D) showed the chest CT of a patient diagnosed with lung cancer: (C) a mixed GGO was found in the left upper lobe; (D) no visible change was detected one month later. GGOs, ground-glass opacities; COVID-19, coronavirus disease 2019.

or nucleic acid negative pneumonia patients (9) and therefore help reduce the missed diagnosis rate and limit the transmission. GGO patients with highly suspected lung cancer should undergo an overall evaluation to exclude COVID-19 infection before the operation. On the one hand, we need to minimize the possibility of COVID-19 being misdiagnosed as lung cancer; alternatively, it has been reported patients with cancer experienced a higher risk of COVID-19 infection and had a poorer prognosis (26). The imprudent decision might expose patients to the attack of surgical trauma and COVID-19 and increase the risk of medical workers as well. Once the diagnosis is confirmed, the priority must be assigned to the treatment of COVID-19 rather than surgery.

Thanks to the rapid and comprehensive response of the Chinese government, public health, medical, and scientific communities, the epidemic control over COVID-19 has been successfully achieved (27). Quarantine measures are gradually relaxed, and more chest CT scans will be performed on regular patients. The importance of discrimination of other pulmonary diseases from
COVID-19, particularly early lung cancer, by CT imaging will be highlighted. In conclusion, the distinctive radiological characteristics of GGOs between COVID-19 and early stage lung cancer, combined with pathogen detection, epidemiological history, laboratory tests, short-term CT reexamination, and pathological results, will benefit the differential diagnosis and lower the rate of missed diagnosis.

\section{Acknowledgments}

Funding: This study was supported by National Natural Science Foundation of China (grant numbers: 81871882, 81902951), Shanghai Municipal Commission of Health and Family Planning Outstanding Academic Leaders Training Program (grant number: 2017BR055), and Shanghai Municipal Education Commission - Gaofeng Clinical Medicine Grant (grant number 20172005).

\section{Footnote}

Reporting Checklist: The authors have completed the 
STROBE reporting checklist. Available at http://dx.doi. org/10.21037/tlcr-20-892

Data Sharing Statement: Available at http://dx.doi. org/10.21037/tlcr-20-892

Conflicts of Interest: All authors have completed the ICMJE uniform disclosure form (available at http://dx.doi. org/10.21037/tlcr-20-892). Dr. YJZ reports grants from National Natural Science Foundation of China, during the conduct of the study. Dr. HCL reports grants from National Natural Science Foundation of China, grants from Shanghai Municipal Commission of Health and Family Planning Outstanding Academic Leaders Training Program, grants from Shanghai Municipal Education Commission Gaofeng Clinical Medicine Grant, during the conduct of the study. The other authors have no conflicts of interest to declare.

Ethical Statement: The authors are accountable for all aspects of the work in ensuring that questions related to the accuracy or integrity of any part of the work are appropriately investigated and resolved. The Ethics Commission of Ruijin Hospital approved this study (KY2020-26) following the Helsinki Declaration (as revised in 2013). Written informed consent for this retrospective study was waived by the Ethics Commission of the designated hospital for emerging infectious diseases.

Open Access Statement: This is an Open Access article distributed in accordance with the Creative Commons Attribution-NonCommercial-NoDerivs 4.0 International License (CC BY-NC-ND 4.0), which permits the noncommercial replication and distribution of the article with the strict proviso that no changes or edits are made and the original work is properly cited (including links to both the formal publication through the relevant DOI and the license). See: https://creativecommons.org/licenses/by-nc-nd/4.0/.

\section{References}

1. World Health Organization. WHO Director-General's statement on IHR Emergency Committee on Novel Coronavirus (2019-nCoV). 2020 [cited 2020 Jun 21]. Available online: https://www.who.int/dg/speeches/detail/ who-director-general-s-statement-on-ihr-emergencycommittee-on-novel-coronavirus-(2019-ncov)

2. World Health Organization. Coronavirus Disease
(COVID-19) Situation Reports. 2020 [cited 2020 Jun 21]. Available online: https://www.who.int/emergencies/ diseases/novel-coronavirus-2019/situation-reports/

3. Mason RJ. Pathogenesis of COVID-19 from a cell biology perspective. Eur Respir J 2020;55:2000607.

4. Pneumonia diagnosis and treatment plan for SARSCoV-2 infection (trial version 7). 2020 [cited 2020 Jun 21]. Available online: http://www.gov.cn/zhengce/ zhengceku/2020-03/04/5486705/files/ae61004f930d47598 711a0d4cbf874a9.pdf

5. Jin YH, Cai L, Cheng ZS, et al. A rapid advice guideline for the diagnosis and treatment of 2019 novel coronavirus (2019-nCoV) infected pneumonia (standard version). Mil Med Res 2020;7:4.

6. Pan F, Ye T, Sun P, et al. Time Course of Lung Changes at Chest CT during Recovery from Coronavirus Disease 2019 (COVID-19). Radiology 2020;295:715-21.

7. Pan Y, Guan H, Zhou S, et al. Initial CT findings and temporal changes in patients with the novel coronavirus pneumonia (2019-nCoV): a study of 63 patients in Wuhan, China. Eur Radiol 2020;30:3306-9.

8. Chung M, Bernheim A, Mei X, et al. CT Imaging Features of 2019 Novel Coronavirus (2019-nCoV). Radiology 2020;295:202-7.

9. Xie X, Zhong Z, Zhao $W$, et al. Chest CT for Typical Coronavirus Disease 2019 (COVID-19) Pneumonia: Relationship to Negative RT-PCR Testing. Radiology 2020;296:E41-5.

10. National Lung Screening Trial Research Team, Church TR, Black WC, et al. Results of initial low-dose computed tomographic screening for lung cancer. N Engl J Med 2013;368:1980-91.

11. Jacobson FL, Jaklitsch MT. Computed Tomography Scanning for Early Detection of Lung Cancer. Annu Rev Med 2018;69:235-45.

12. Tian S, Hu W, Niu L, et al. Pulmonary Pathology of Early-Phase 2019 Novel Coronavirus (COVID-19) Pneumonia in Two Patients With Lung Cancer. J Thorac Oncol 2020;15:700-4.

13. Huang P, Liu T, Huang L, et al. Use of Chest CT in Combination with Negative RT-PCR Assay for the 2019 Novel Coronavirus but High Clinical Suspicion. Radiology 2020;295:22-3.

14. Fang Y, Zhang H, Xie J, et al. Sensitivity of Chest CT for COVID-19: Comparison to RT-PCR. Radiology 2020;296:E115-7.

15. Cheng TY, Cramb SM, Baade PD, Youlden DR, Nwogu C, Reid ME. The International Epidemiology 
of Lung Cancer: Latest Trends, Disparities, and Tumor Characteristics. J Thorac Oncol 2016;11:1653-71.

16. Kanne JP. Chest CT Findings in 2019 Novel Coronavirus (2019-nCoV) Infections from Wuhan, China: Key Points for the Radiologist. Radiology 2020;295:16-7.

17. Kim TJ, Goo JM, Lee KW, et al. Clinical, pathological and thin-section CT features of persistent multiple groundglass opacity nodules: comparison with solitary groundglass opacity nodule. Lung Cancer 2009;64:171-8.

18. MacMahon H, Naidich DP, Goo JM, et al. Guidelines for Management of Incidental Pulmonary Nodules Detected on CT Images: From the Fleischner Society 2017. Radiology 2017;284:228-43.

19. Bernheim A, Mei X, Huang M, et al. Chest CT Findings in Coronavirus Disease-19 (COVID-19): Relationship to Duration of Infection. Radiology 2020;295:200463.

20. Kinsey CM, Estepar RS, Zhao Y, et al. Invasive adenocarcinoma of the lung is associated with the upper lung regions. Lung Cancer 2014;84:145-50.

21. Walter JE, Heuvelmans MA, de Jong PA, et al. Occurrence and lung cancer probability of new solid nodules at incidence screening with low-dose CT: analysis of data from the randomised, controlled NELSON trial. Lancet
Oncol 2016;17:907-16.

22. Song YS, Park CM, Park SJ, et al. Volume and mass doubling times of persistent pulmonary subsolid nodules detected in patients without known malignancy. Radiology 2014;273:276-84.

23. Chang D, Lin M, Wei L, et al. Epidemiologic and Clinical Characteristics of Novel Coronavirus Infections Involving 13 Patients Outside Wuhan, China. JAMA 2020;323:1092-3.

24. Chen N, Zhou M, Dong X, et al. Epidemiological and clinical characteristics of 99 cases of 2019 novel coronavirus pneumonia in Wuhan, China: a descriptive study. Lancet 2020;395:507-13.

25. Ng MY, Lee EY, Yang J, et al. Imaging Profile of the COVID-19 Infection: Radiologic Findings and Literature Review. Radiol Cardiothorac Imaging 2020;2:e200034.

26. Liang W, Guan W, Chen R, et al. Cancer patients in SARS-CoV-2 infection: a nationwide analysis in China. Lancet Oncol 2020;21:335-7.

27. China National Health Commission. Chinese mainland sees 20 provincial regions clear of COVID-19 for over 28 days. 2020 [cited 2020 Jun 21]. Available online: http:// en.nhc.gov.cn/2020-03/29/c_78448.htm
Cite this article as: Zhang YJ, Yang WJ, Liu D, Cao YQ, Zheng YY, Han YC, Jin RS, Han Y, Wang XY, Pan AS, Dai JY, Sun QF, Zhao FQ, Yang QY, Zhang JH, Liu SJ, Da Q, Guo W, Li CQ, Zhang WT, Wu H, Chen XS, Ji AQ, Xiang J, Chen K, Feng XJ, Zhang XF, Cao QQ, Qin L, Li J, Zhou M, Lu Y, Wang CF, Yan FH, Li HC, Qu JM. COVID-19 and early-stage lung cancer both featuring ground-glass opacities: a propensity score-matched study. Transl Lung Cancer Res 2020;9(4):1516-1527. doi: $10.21037 /$ tlcr-20-892 

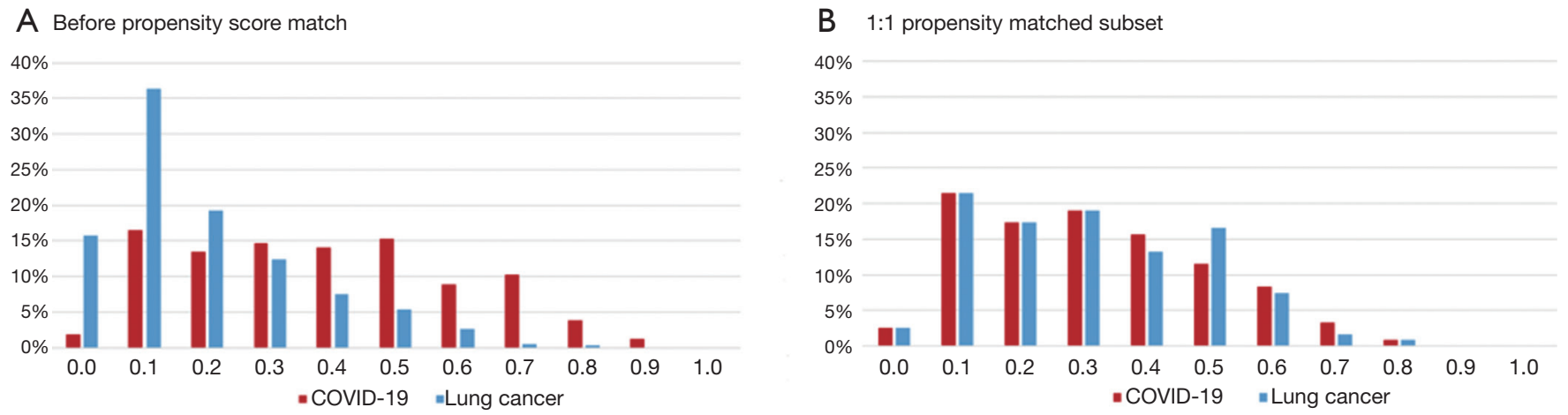

Figure S1 Distribution of propensity score.

Table S1 Location and type of lung lesions

\begin{tabular}{|c|c|c|c|}
\hline Variables & COVID-19 ( $n=1,207$ lesions) & Lung cancer ( $\mathrm{n}=448$ lesions) & $P$ value \\
\hline Peripheral & $923(76 \%)$ & $333(74 \%)$ & 0.40 \\
\hline Central & $55(5 \%)$ & $29(6 \%)$ & 0.15 \\
\hline Both & $209(17 \%)$ & $86(19 \%)$ & 0.42 \\
\hline Type of lesions, $n$ (\% of all lesions) & & & $<0.0001^{*}$ \\
\hline Pure GGO & $321(27 \%)$ & $204(46 \%)$ & $<0.0001$ \\
\hline Mixed GGO & $695(58 \%)$ & $210(47 \%)$ & 0.00013 \\
\hline Consolidation & $170(14 \%)$ & $24(5 \%)$ & $<0.0001$ \\
\hline
\end{tabular}

*, Pearson's chi-squared test for overall distribution. GGO, ground-glass opacity.

COVID-19
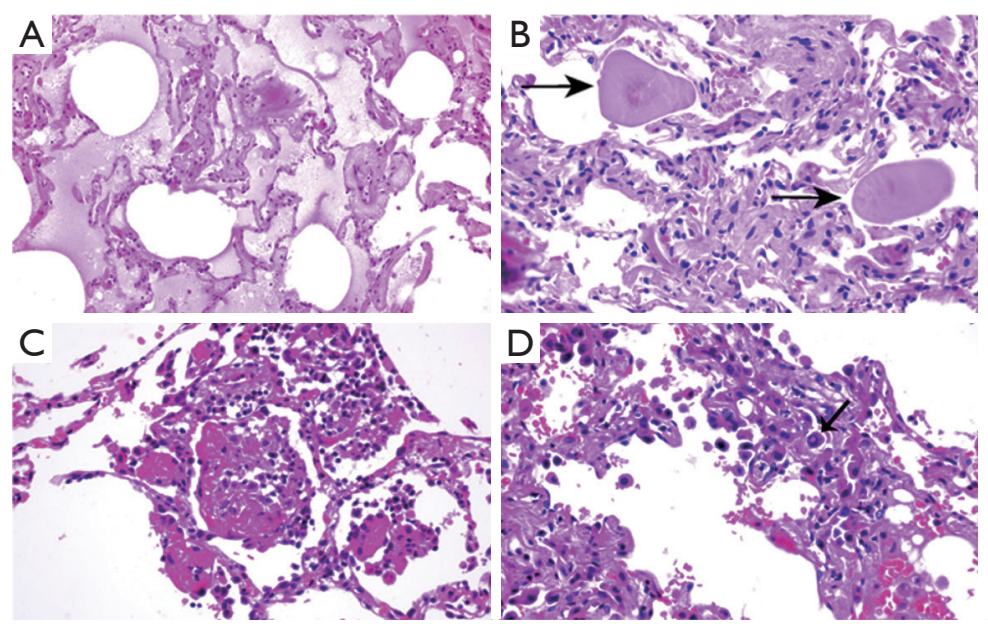

Early stage adenocarcinoma
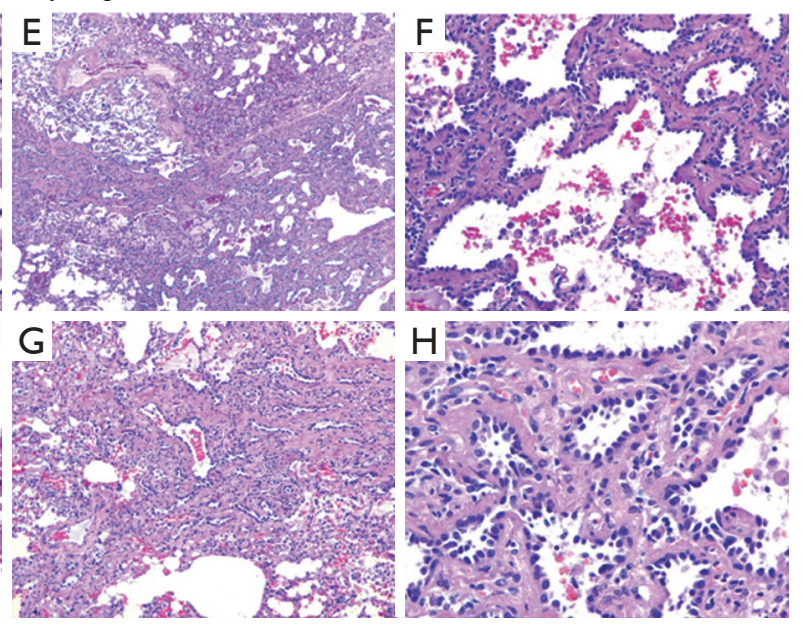

Figure S2 Pathological results of COVID-19 (A,B,C,D) ${ }^{1}$ and early stage lung cancer (E,F,G,H). Scale: 1:200. COVID-19, coronavirus disease 2019. ${ }^{1}$, reproduced by permission of the owner of the publishing rights of Reference 12 . 